\title{
Liquid Metal Enabled Unconventional Heat and Flow Transfer
}

\author{
Sen Chen ${ }^{1,2,4}$ and Jing $\operatorname{Liu}^{1,2,34^{*}}$
}

\begin{abstract}
Ga-based liquid metal has obtained great attention because of its excellent physical and chemical properties, such as extraordinary thermal conductivity, high electrical conductivity, excellent fluidity. Thus, liquid metal has increasingly been used in many applications, including the microfluidics, biomedical applications, flexible electronics, soft machines and thermal management. Significant progress in science and industry has been made in the past two decades about the Ga-based liquid metal. Among them, the progress in the field of heat transfer deserves our attention because of its huge industrial value. Many cooling methods based on liquid metal have been developed to accommodate the urgent needs to solve extreme heat flow problems. In this review, we first give an overview of the uniqueness of liquid metal as an untraditional cooling medium. Then strategies to improve the properties of Ga-based liquid metal are presented. The corresponding basic theory of liquid metal heat transfer is discussed. In the subsequent sections, the applications of liquid metal as a fluid in convective heat transfer will be reviewed in details. Finally, key scientific and technical issues and challenges in liquid metal heat transfer will be pointed out to guide future practices.
\end{abstract}

Keywords: Gallium-based liquid metal; Thermal management; Fluid properties; Modification strategy; Convection Heat Transfer

Received 30 May 2019, Accepted 20 July 2019

DOI: $10.30919 / \mathrm{esee} 8 \mathrm{c} 318$

\section{Introduction}

With the rapid development of the electronics and the semiconductor industry, the power of the chip of electronic devices is gradually increasing. ${ }^{1}$ During the working process of the chip, part of the power will turn into heat, which is inevitable according to the second law of thermodynamics. As a result, the "thermal barrier" problem has become an important challenge hindering the development of electronic chips. It is extremely urgent to develop high-performance chip cooling and thermal management methods. Analogously, the needs for heat dissipation are also present in more occasions. To meet the cooling needs of the electronics industry, many strategies are applied to heat dissipation. Among the strategies ever established, air cooling, 2,3 including natural convection and forced convection, which is limited by its poor cooling capacity and is generally used in locations with a heat flux density of less than $10 \mathrm{~W} / \mathrm{cm}^{2}$. Heat pipe is an excellent heat dissipation method for long-distance heat transfer with low temperature difference, which has been widely used on chips having a heat flux density of several tens of W/cm ${ }^{2} ., 5$ For higher heat flux, such as $10^{2}$ $\mathrm{W} / \mathrm{cm}^{2}$ even $10^{3} \mathrm{~W} / \mathrm{cm}^{2}$, the performance of heat pipe with complex structure is usually unstable.

At this case, liquid cooling becomes a viable choice, ${ }^{6}$ including

${ }^{1}$ Beijing Key Lab of Cryo-Biomedical Engineering, Key Lab of Cryogenics, Technical Institute of Physics and Chemistry, Chinese Academy of Sciences, Beijing 100190, China

${ }^{2}$ School of Future Technology, University of Chinese Academy of Sciences, Beijing 100049, China

${ }^{3}$ Key Lab of Cryogenics, Technical Institute of Physics and Chemistry, Chinese Academy of Sciences, Beijing 100190, China

${ }^{4}$ Department of Biomedical Engineering, School of Medicine, Tsinghua

University, Beijing 100084, China

*E-mail: jliu@mail.ipc.ac.cn micro-channel, ${ }^{7}$ two phase convection cooling, ${ }^{8}$ spray cooling ${ }^{9}$ etc. Generally, the heat dissipating medium used in liquid cooling is water, thereby leading to the result that convection cooling still cannot meet the needs for extreme heat dissipation. Therefore, other fluids with better heat dissipation effect are needed. Liquid metal convection cooling technology emerges as the times require. Since 2002, Liu's group introduced the room temperature Ga-based liquid metal into the computer chip cooling for the first time, ${ }^{10,11}$ which largely promotes the research and application of the Ga-based liquid metal in the field of heat transfer. From then on, more theoretical and experimental studies on Ga-based liquid metal cooling technology have been carried out and gained continuous attention. ${ }^{12-14}$ As a new class of thermal and energy management materials, Ga-based liquid metal brings great ideas and technical innovations in the field of heat transfer, providing a new solution for heat transfer. Therefore, Ga-based liquid metal is expected to break the performance limit of traditional cooling technology. Actually, as early as the last century, liquid metal represented by leadbased alloys has been applied in the field of heat dissipation. In 1963, the lead-based alloys were used to cool the nuclear-power submarine. ${ }^{15}$ In addition to that, sodium, mercury, and $\mathrm{NaK}$ alloys were also applied to cool the heat source (e.g. nuclear reactor). ${ }^{16,17}$ However, the leadbismuth coolant is generally corrosive for structural materials and easily contaminates the wall. Sodium, mercury, and $\mathrm{NaK}$ alloys are unsafe due to the flammability or toxicity.

As a result, non-toxic, non-flammable Ga-based liquid metal attracts extensive attention in the civilian sector. Currently, the progress of Ga-based liquid metal in the field of heat transfer is impressive because of its application prospects." Many heat transfer strategies based on Ga-based liquid metal have therefore been developed, including low-melting phase change materials, thermal interface materials, convective cooling and new cooling strategies. ${ }^{18-20}$ In addition to that, Ga-based liquid metal has been used in many areas, including soft electronics, ${ }^{21-25}$ biomedical applications, ${ }^{26-30}$ chemical synthesis and 
catalysis, ${ }^{31-34}$ and soft machines. ${ }^{35-38}$ The reason lying behind the widespread application is mainly due to its excellent properties, including high electricity, high thermal conductivity, low vapor pressure, high boiling point, excellent liquidity and biological compatibility. ${ }^{3943}$ More importantly, many studies have proven that Ga-based liquid metal is non-toxic and biocompatible compared to the well-known mercury. ${ }^{4450}$ Therefore, it is of great scientific and practical value to summarize and comb the recent progress of Ga-based liquid metal.

In this paper, we focus on the fluid properties of Ga-based liquid metal and their modification methods to better guide the applications of Ga-based liquid metal in the field of heat transfer, especially for the applications in convection heat dissipation. Ga-based liquid metal, as a non-traditional fluid, exhibits unique properties. Herein, we summarize the fluid properties of Ga-based liquid metal. It is worth mentioning that the properties of Ga-based liquid metal can be well modified. Therefore, strategies to improve the properties of Ga-based liquid metal are listed. More clearly, some basic heat transfer theory and typical applications of Ga-based liquid metal have also been demonstrated. Finally, key scientific and technical issues and challenges in liquid metal fluid science and heat transfer will be presented.

\section{Fluid properties of liquid metal and improvement strategies}

\subsection{Basic fluid properties of Ga-based liquid metal}

To better promote the practical applications of Ga-based liquid metal, it is necessary to deeply understand the fluid properties of Ga-based liquid metal. Compared to the water, a common fluid medium, the thermal conductivity of Ga-based liquid metal is almost dozens of times than that of water, which is considered to be the most significant and important thermal property of Ga-based liquid metal. As for the viscosity, the viscosity of Ga-based liquid metal is approximately twice than that of water, indicating that it has excellent fluidity. Besides, Gabased liquid metal belongs to the metal category, which guarantees its metal properties, including high electrical conductivity and large surface tension. Unlike traditional non-conductive fluids, Ga-based liquid metal benefits from the excellent electrical conductivity, allowing it to flexibly change its properties by applying an electric field. Specifically, the comparison of the thermos-physical properties between water and liquid metal is listed in the Table 1.

To our best knowledge, the surface tension of Ga-based liquid metal is the largest in all the room temperature fluid, which is almost ten times than that of water. ${ }^{39}$ Analysis of the electric capillary curve of liquid metal reveals that a small applied voltage (below $5 \mathrm{~V}$ ) can quickly change the surface tension of Ga-based liquid metal, and the surface tension of the fluid has a great influence on the fluid behaviors of Ga-based liquid metal. Therefore, many fascinating studies have been carried out based on the largest surface tension. ${ }^{36-38,53-58}$ Exploring these phenomena is critical to understanding the hydrodynamic behaviors of Ga-based liquid metal. More importantly, these fascinating studies involve the driving of Ga-based liquid metal, such as electric field drive, ${ }^{58,59}$ self-driven. ${ }^{36,38,60}$ Such special drive strategies can be used to drive liquid metal for heat dissipation, which has been achieved by a series of researches. ${ }^{61-63}$

For a more intuitive understanding of the hydrodynamic properties of Ga-based liquid metal, the Faraday wave effect of Ga-based liquid metal is introduced as a typical example. Metallic fluids may contain rather rich unconventional surface wave behaviors when subjected to specific forcing conditions. The liquid rises up and down vertically with the container, and a non-linear surface standing wave is formed on the surface of the liquid, which is so-called a Faraday wave, a typical hydrodynamic instability phenomenon. Generally, the fluids involved in former studies are primarily water and oils that are relatively small in density and surface tension. Besides, such fluids cannot be manipulated by an electric field because of its poor conductivity. To demonstrate the uniqueness of the fluid properties of Ga-based liquid metal, researchers investigated the high-surface-tension liquid metal-solution bilayer to the Faraday system and its wave evolution under cylindrical confinement. ${ }^{64}$

In this study carried by Zhao et $a l^{64}$ beyond the Faraday threshold, it was observed the formation of highly symmetric wave patterns.

Table 1 Comparison of the thermos-physical properties between water and liquid metals.

\begin{tabular}{|c|c|c|c|c|c|}
\hline Materials & $\begin{array}{l}\text { Density } \\
\rho\left(\mathrm{kg} / \mathrm{m}^{3}\right)\end{array}$ & $\begin{array}{l}\text { Heat } \\
\text { capacity }\end{array}$ & $\begin{array}{l}\text { Viscosity } \mu \\
\left(10^{-3} \mathrm{~kg} / \mathrm{m} \cdot \mathrm{s}\right)\end{array}$ & $\begin{array}{l}\text { Thermal } \\
\text { conductivity }\end{array}$ & $\begin{array}{l}\text { Melting } \\
\text { point }\end{array}$ \\
\hline & & $c_{p}(\mathrm{~J} / \mathrm{kg} \cdot \mathrm{K})$ & & $k(\mathrm{~W} / \mathrm{m} \cdot \mathrm{K})$ & $\operatorname{Tm}\left({ }^{\circ} \mathrm{C}\right)$ \\
\hline Water* & 998 & 4182 & 1.003 & 0.6 & 0 \\
\hline $\mathrm{Hg}$ & 13564 & 139 & 1.56 & 8.7 & -38.9 \\
\hline $\mathrm{Na}_{27} \mathrm{~K}_{73} 51$ & 868 & 982 & 0.91 & 21.8 & -11 \\
\hline $\mathrm{Ga}_{61} \mathrm{In}_{25} \mathrm{Sn}_{13} \mathrm{Zn}_{1}{ }^{11}$ & 6500 & - & - & 15 & 7.6 \\
\hline $\mathrm{Ga}_{68} \mathrm{In}_{20} \mathrm{Sn}_{12}{ }^{12}$ & 6363 & 366 & 2.22 & 39 & 10.7 \\
\hline $\mathrm{Ga}_{80} \mathrm{In}_{20}$ & 6335 & 404 & - & 26.6 & 16 \\
\hline $\mathrm{Ga}^{52}$ & 6095 & 398 & 1.75 & 33.7 & 29.8 \\
\hline Cs & 1879 & 240 & - & 35.9 & 28.5 \\
\hline
\end{tabular}

*At $20{ }^{\circ} \mathrm{C}$. 
Researchers further show that non-coalescent droplets of the same liquid metal can be sustained on the structured wave patterns. Distant droplets can be confined at the antinodes of the wavy surface and selfassemble into the corresponding wave structures (Fig. 1a). For conventional fluids, the study of suspended droplets can only be limited to critical Faraday accelerations. Once the drive acceleration is above the Faraday threshold, the entire surface will suddenly become turbulent and no longer carry suspended droplets. Since the Ga-based liquid metal system can form a highly regular pattern, the metal droplets can be stably suspended on the liquid surface even at a critical acceleration above the Faraday threshold.

These series of highly symmetrical surface wave patterns excited on the surface of Ga-based liquid metal have never been observed in a single conventional fluid system. The main reason is that the large surface tension of the Ga-based liquid metal causes the dissipation length to be much higher than that of the conventional fluid. More importantly, the surface state of Ga-based liquid metal can be flexibly and rapidly regulated by an applied electric field, thus the researchers demonstrate a simple yet fast method to switch the surface waves between different states by electrically altering the liquid metal surface tension. Here, one can see that surface tension plays a vital role in affecting the wave behaviors. Finally, it is worth noting that adjusting the surface wave mode by an applied electric field is a reversible process. When the applied electric field is removed, the surface wave can automatically return to its original state. Therefore, it can be seen that the Ga-based liquid metal having a large surface tension is a kind of fluid with excellent fluidity and can be regulated by an electric field.
Based on these properties, more applications of Ga-based liquid metal in the field of heat transfer can be carried out.

Because of its large surface tension, many novel hydrodynamic behaviors occur. Another question worth pondering is whether Ga-based liquid metal that are different from traditional liquids belong to NonNewtonian fluids. Here, the so-called Non-Newtonian fluid refers to a fluid that does not meet the Newtonian viscosity test law, that is, a fluid whose shear stress and shear strain rate are not linear. Non-Newtonian fluids are widely found in life and nature. Most biological fluids belong to the non-Newtonian fluids now defined. As for the Newtonian fluid we are familiar with, the most typical one is water. In recent years, researches show that a linear relationship between the viscous stress and the strain rate at any point exists in the liquid metal fluid, thus we can initially conclude that Ga-based liquid metal can be thought as Newtonian fluid. ${ }^{65}{ }^{66}$ However, the Ga-based liquid metal with changed viscosity will display some non-Newtonian fluid behaviors, ${ }^{67}$ which should be considered in certain applications. Here, oxidation is an important factor affecting the viscosity. Researches show that Ga-based liquid metal will be easily oxidized when it is exposed to the air. ${ }^{68,69}$ Further, the properties of oxide layer of Ga-based liquid metal are totally different from the pure Ga-based liquid metal and the mechanical properties of Ga-based liquid meal can be finely and reversibly controlled by adjusting the degree of oxidation. ${ }^{70}$ Therefore, oxidation will significantly affect the properties of Ga-based liquid metal.

\subsection{Strategies to improve the properties of Ga-based liquid metal}

Even if Ga-based liquid metal displays excellent properties, it is also a
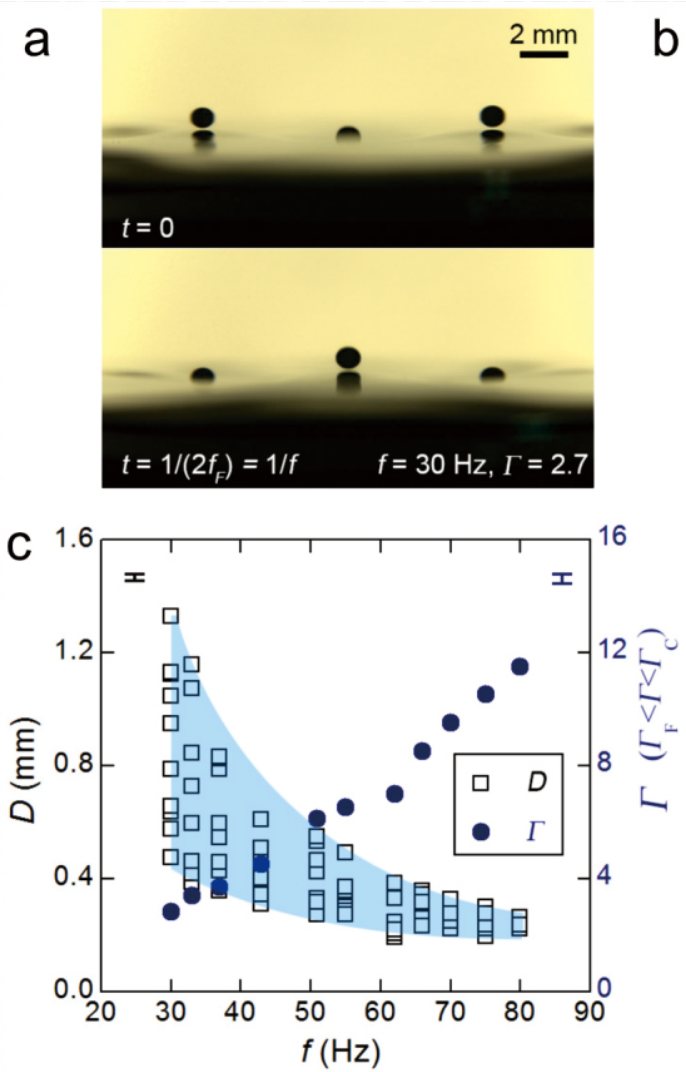

b
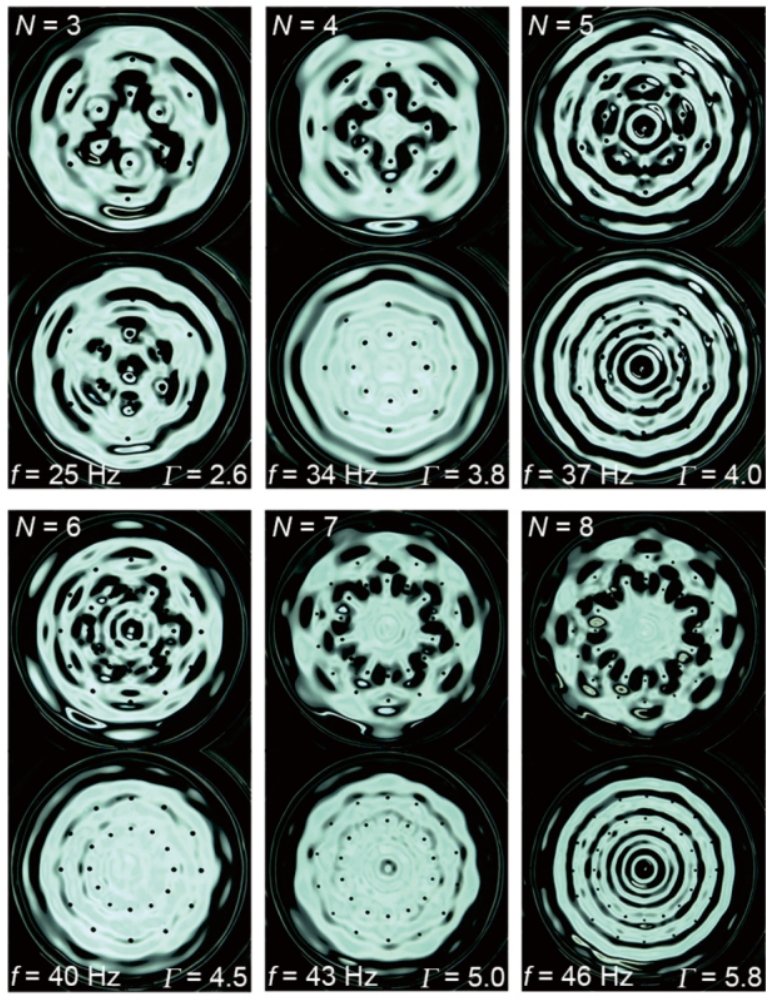

Fig. 1 Bouncing droplets on the vibrating Ga-based liquid metal bath. (a) Droplets being locked at the wave antinodes and adjacent droplets bouncing in antiphase at the Faraday frequency; (b) Self-assemblies of liquid metal droplets at their Faraday bouncing state when the bath is vibrating with different fold symmetry number $N$; (c) change of the diameter range of Faraday bouncing droplets that can be sustained as a function of $f$. The area filled in blue indicates the Faraday bouncing region. @ copyright from Ref. 64 with permission from American Physical Society. 
important to further modify its properties to fulfill more special needs. For example, the specific heat capacity of Ga-based liquid metal is still somewhat small and it is meaningful to increase the specific heat capacity of Ga-based liquid metal through adding the particles with high specific heat capacity. Fortunately, Ga-based liquid metal is naturally liquid, so its properties can be easily tuned by oxidation or doping. As stated above, oxidation will change the properties of Ga-based liquid metal, thus we can tune the properties of Ga-based liquid metal by controlling the degree of oxidation. In addition to that, actively doping the particles into the Ga-based liquid metal is a more important and controllable strategy of modifying the corresponding properties. Herein, the methods of doping are classified and summarized to guide future practices.

To dope the required substances into Ga-based liquid metal, continuous mixing is an obvious strategy. In this regard, related efforts and progress have been achieved. As illustrated in Fig. 2, Cu, Fe, Mg, $\mathrm{Ni}$, and Gd are all successfully mixed with Ga-based liquid metal. Actually, because of the huge surface tension of Ga-based liquid metal, such mixing is not easy to succeed and taking the corresponding experimental skills is necessary. Specifically, Ga-based liquid metal can be well mixed with the copper powder by directly stirring at room temperature (Fig. 2a). The reason lying behind this is that there is good wetting between Ga-based liquid metal and copper. ${ }^{71}$ During the process, $\mathrm{Cu}-\mathrm{Ga}$ alloy was formed, which has been confirmed by micrograph (Fig. 2b). Differently, $\mathrm{Mg}$ powder and Ga-based liquid metal need to be heated to $300{ }^{\circ} \mathrm{C}$ to get a good mixing effect (Fig. 2c). Meanwhile, the high temperature ensures the formation of Mg-Ga twophase alloy. $^{72}$ For $\mathrm{Fe}$ power, $\mathrm{HCl}$ solution was added to remove the oxide layer on the surfaces of the iron particles and Ga-based liquid metal, making it easier for iron particles to be absorbed (Fig. 2d). ${ }^{73}$ The modified Ga-based liquid metal obtained by this strategy still maintains the state of the fluid. Besides, the obtained Ga-based liquid metal is magnetic and can be controlled by a magnetic field, ${ }^{74}$ which is beneficial for the future applications.

Further, the study found that oxidation also contributes to the doping between Ga-based liquid metal and Ni particles. ${ }^{75}$ As shown in Fig. 2e, by continuously stirring the Ga-based liquid metal and $\mathrm{Ni}$ powder mixture, the mixture was divided into two layers, one layer consisting of only Ga-based liquid metal (EGaIn layer), and the other layer consisting of a mixture of liquid metal and nickel powder (GIN layer). Here, the viscosity of the oxidized Ga-based liquid metal is greatly increased, so that the Ni powder can be adhered and then absorbed into the Ga-based liquid metal. It should be noted that Gabased liquid metal modified in this way no longer maintains the fluid state, which contributes to the application as thermal interfaces materials. Besides, gadolinium also can be added into the liquid metal for obtaining additional magnetic properties. Here, gallium-based liquid metal alloy is used as the solvent and suspension medium. As shown in Fig. 2f, Gd/galinstan ferrofluid consists of liquid metal and $\mathrm{Gd}$ nanoparticles. With the increase of the metals temperature caused by the exposure of the Gd nanoparticles to a magnetic field. the energy is consequently released to the environment as heat. Then, when the ferrofluid leaves the magnetic field, it features a temperature lower than that in step I. Therefore, the $\mathrm{Gd} /$ galinstan ferrofluid can be used to magnetocaloric cooling.

Moreover, the properties of Ga-based liquid metal can be modified by utilizing the physical effects associated with liquid metal. A previously reported study proposed a way called LM-Phagocytosis to add metal particles to the interior of Ga-based liquid metal, ${ }^{72}$ which is effective and important for improving the thermal and fluid properties of Ga-based liquid metal. As illustrated in Fig. 3, the $\mathrm{Cu}$ particles can be swallowed into the Ga-based liquid metal, thereby achieving the doping between metal particles and Ga-based liquid metal. To achieve this, a prerequisite for the particles to enter the interior of Ga-based

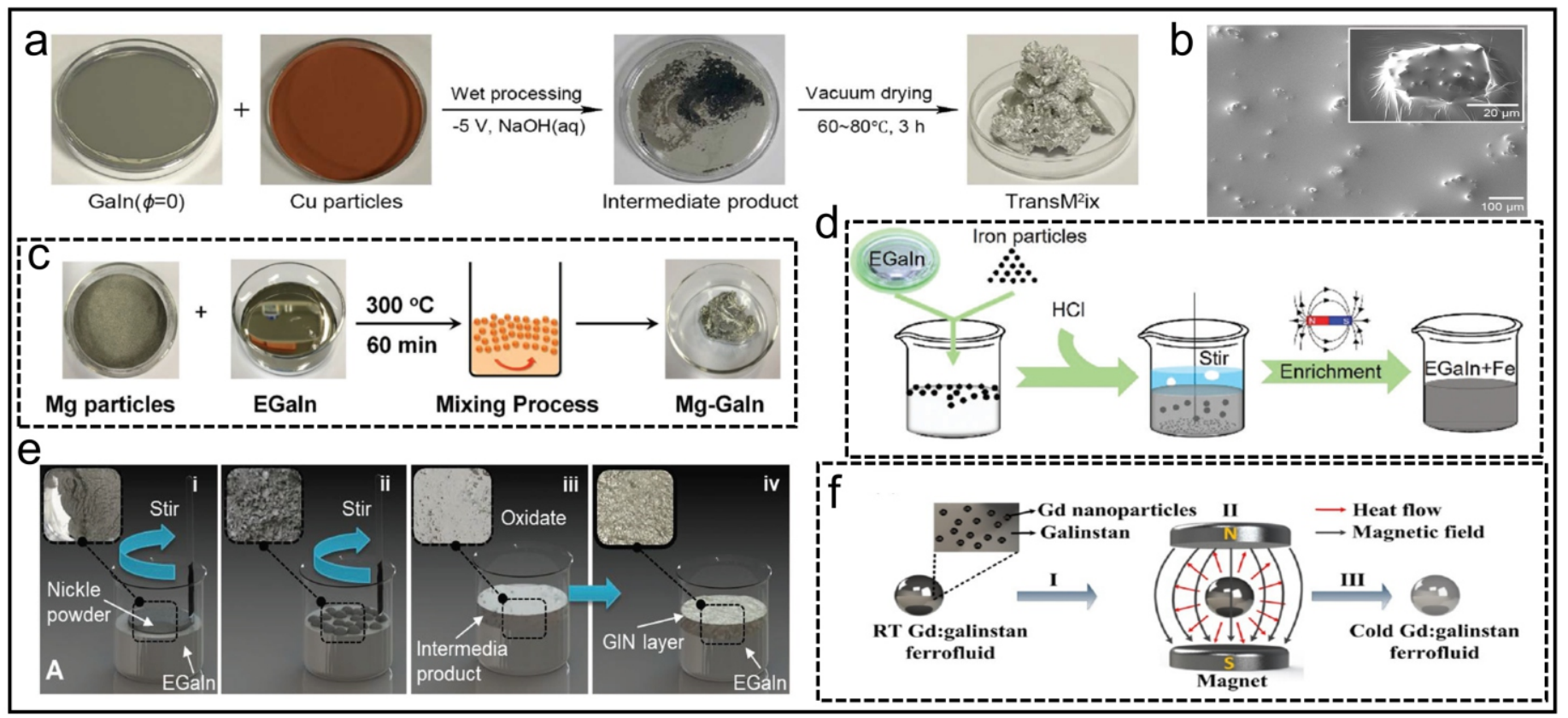

Fig. 2 Methods to realize modified liquid metal by doping other metals into liquid metal: a) Cu-doped Ga-based liquid metal; ${ }^{71}$ Reproduced from Ref. 71, with permission from American Chemical Society. b) Micro-picture of Cu-doped Ga-based liquid metal; Reproduced from Ref. 71, with permission from American Chemical Society. c) Mg-doped Ga-based liquid metal; Reproduced from Ref. 72, with permission from Wiley-VCH. d) Fe-doped Gabased liquid metal: PLUS materials; ${ }^{73}$ Reproduced from Ref. 73 with permission from The Royal Society of Chemistry. e) Schematic illustration of the stage for the synthesis process of the GIN materials, ${ }^{75}$ Reproduced from Ref. 75 , with permission from Wiley-VCH. f). Schematic of the magnetocaloric cooling process based on our developed magnetic liquid metal. ${ }^{19}$ Reproduced from Ref. 19, with permission from American Chemical Society. 
liquid metal is the remove of the oxide film which is present at both the interface of $\mathrm{Ga}$-based liquid metal and $\mathrm{Cu}$ particles. In this regard, the researchers proposed three types of incentives to achieve the LMPhagocytosis that Ga-based liquid metal swallowed the copper particles, namely: electrocautery polarization, auxiliary metal polarization and chemical triggering. Besides, the work revealed the law of such physical effect of Ga-based liquid metal in acidic, alkaline and neutral solution environments. Among them, the method of generating cathode polarization by applying an electric field owns the advantages of being quick and controllable, easy to operate. Further research has also found that the mechanism governing the LM-Phagocytosis is the wetting between the solid/liquid two metal phases. The researchers have established a general rule for quantitatively characterizing the contact relationship between the solid/liquid two metal phases. The theoretical model better explains the experimental results and estimates the energy barrier of the particle endocytosis of different materials, further predicting whether the LM-Phagocytosis can proceed spontaneously. After the process, the properties of Ga-based liquid metal can be well adjusted. According to this model, more particles can also be swallowed by Ga-based liquid metal, thereby achieving the goal of regulating its physical properties.

Actually, the nature of doping metal particles to change the properties of Ga-based liquid metal belongs to local alloying. Such local alloying contributes to form a metal solid solution or a metal compound, thereby altering the physical properties of Ga-based liquid metal. Further, whether more other non-metallic particles can be doped into the Ga-based liquid metal to tune the properties (the viscosity, thermal conductivity and specific heat capacity) is equally worth

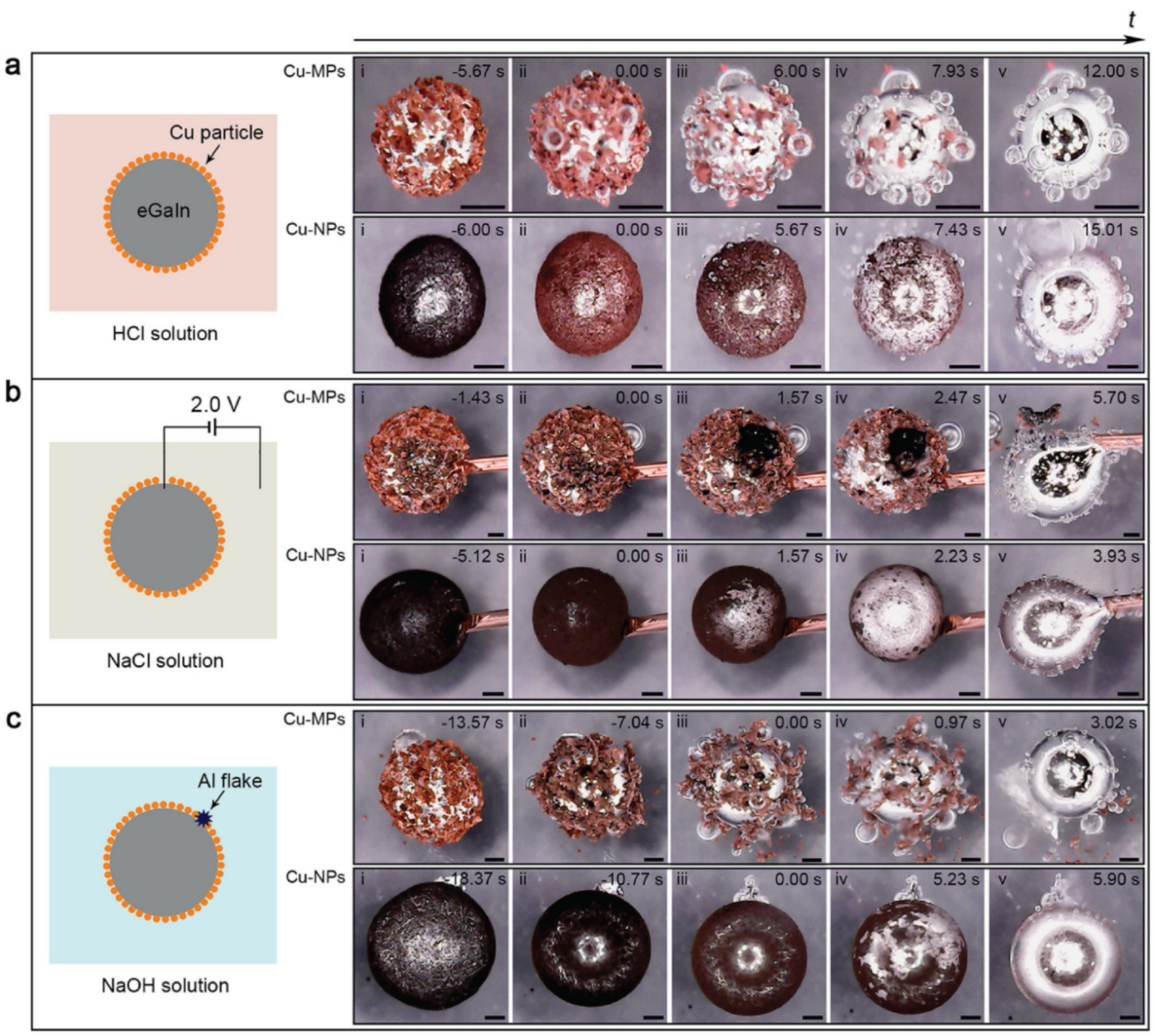

Fig. 3 Methods to realize LM-Phagocytosis in different solutions: a) Acidic solution with no additional assistance. b) Neutral solution assisted by an electrical polarization; c) Alkaline solution assisted by an aluminum flake. Here, $\mathrm{Cu}-\mathrm{MPs}$ and $\mathrm{Cu}-\mathrm{NPs}$ mean $\mathrm{Cu}$ micro-particles and $\mathrm{Cu}$ nano-particles. Scale bars: $200 \mu \mathrm{m}^{72}$ Reproduced from Ref. 72, with permission from Wiley-VCH. 
thinking and trying. Along this way, more trials and efforts need to be implemented.

\section{Basic theory of liquid metal heat transfer}

Ga-based liquid metal is an unconventional fluid because of the unique properties. ${ }^{76}$ Such unconventional properties bring about heat transfer characteristics different from common fluids (e. g. water). Therefore, introducing the basic theory of Ga-based liquid metal heat transfer will be necessary for practicing the application.

Based on the fluid properties of Ga-based liquid metal, this review mainly discusses the convective heat dissipation. For convection heat transfer, Newton's cooling law is as follows:

$$
q=h \Delta T_{C}
$$

where $q$ is flux, $h$ is convective heat transfer coefficient and $\Delta T_{C}$ is characteristic temperature difference. From the Newtonian cooling equation, we can clearly see that the cooling effect is influenced by convective heat transfer coefficient and characteristic temperature difference. Generally, characteristic temperature difference is definite. Therefore, increasing the convection heat dissipation coefficient is the top priority for improving convection cooling capacity.

The dimensionless parameter Nusselt number $\mathrm{Nu}$ is usually used to evaluate the heat transfer intensity, which is defined as follows:

$$
N u=\frac{h D}{k}
$$

where $D$ is the characteristic length and $k$ is the thermal conductivity of the fluid. Table 2 summarizes the correlations of $N u$ for fully developed convective heat transfer in a circular pipe under constant temperature or constant heat flux condition. In which, Reynolds number $R e$ is an important dimensionless parameter in fluid mechanics, which is defined as the ratio of inertial force to viscous force:

$$
R e=\frac{\rho U D}{\mu}
$$

where, $\rho$ and $\mu$ are density and viscosity of the fluid, $U$ is characteristic flow velocity. The Prandtl number $P r$ is another important dimensionless number in heat transfer problem, which is the ratio of momentum diffusivity to thermal diffusivity:

$$
\operatorname{Pr}=\frac{v}{\alpha}
$$

where $v=\mu / \rho$ and $\alpha=\mu / \rho, c_{p}$ is specific heat capacity.

The $P r$ number of different fluids varied a lot. In general, the $\mathrm{Pr}$ number of air, water, oil and glycerol is about $0.7,7$ (at $20{ }^{\circ} \mathrm{C}$ ), 10 and 1000 , respectively. ${ }^{77}$ However, for the liquid metal, $P r$ is really small (about $10^{-2}$ magnitude), which is mainly due to the large thermal conductivity. The small $\mathrm{Pr}$ number means that the temperature transfer rate of Ga-based liquid metal is rather fast, which causes the temperature boundary layer to expand rapidly, in turn affecting the middle portion of the Ga-based liquid metal flow. Therefore, for liquid metal convection heat dissipation, its axial heat conduction is not negligible. For concreteness, the convective heat transfer correlations for water and liquid metal in circular pipe are listed as followed.

Such characteristics indicate that Ga-based liquid metal has significant advantages in convective heat transfer. In order to clearly show this advantage, researchers compared the performance of Gabased liquid metal and water in the convection of a circular tube." Here, the inner and outer diameters of the tube are $5 \mathrm{~mm}$ and $7 \mathrm{~mm}$, respectively. The flow rates of Ga-based liquid metal and water are set to $0.5 \mathrm{~m} / \mathrm{s}$, and the initial inlet temperature is set to $20{ }^{\circ} \mathrm{C}$. In addition, such tube is uniformly heated from the outer wall with constant heat flux of $20 \mathrm{~W} / \mathrm{cm}^{2}$. The results of the simulation are shown in the Fig. 4. For water cooling, the temperature of the round tube will reach $130{ }^{\circ} \mathrm{C}$

(a) Water cooling

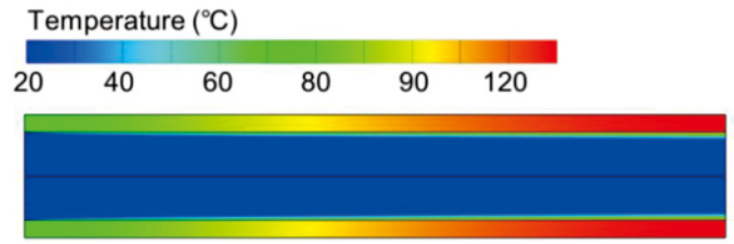

(b) Liquid metal cooling
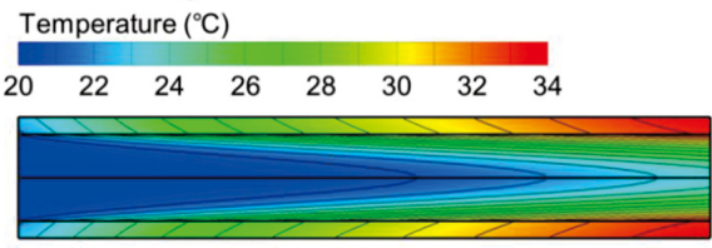

Fig. 4 Intuitive comparison of water cooling and Ga-based liquid metal cooling in a circular pipe under constant heat flux. ${ }^{11}$ Reproduced from Ref. 11, with permission from Elsevier.

Table 2 Convective heat transfer correlations for water and liquid metal in circular pipe. .1,78 $^{5}$

\begin{tabular}{llll}
\hline Coolant & Flow regime & Constant heat flux & Constant wall temperature \\
\hline \multirow{2}{*}{ Water } & Laminar flow & $N u=4.364$ & $N u=3.657$ \\
& Turbulent flow & $N u=0.023 \operatorname{Re}^{0.8} \mathrm{Pr}^{\mathrm{n}} n=0.4$ for heating, $n=0.3$ for cooling \\
& Laminar flow & $N u=4.364$ & $N u=3.657+4.487 / \mathrm{Pe}^{2}$ \\
Liquid metal & & & $P e>5^{*}$ \\
& Turbulent flow & $N u=4.82+0.0185 \mathrm{Pe}^{0.827}$ & $N u=5.0+0.025 \mathrm{Pe}^{0.8}$ \\
\hline
\end{tabular}

$* P e=\operatorname{RePr}$ 
However, for the case of the Ga-based liquid metal as a convection medium, the temperature of the tube can be well controlled (below $34{ }^{\circ} \mathrm{C}$ ). The reason lying behind it is that the thin boundary layer in the water cooling process limits the heat transfer, which makes it difficult for the remaining bulk water to cool the wall. For the liquid metal, heat can be quickly transferred to the interior of the fluid and then absorbed efficiently because of the inherently large thermal conductivity, thus resulting in an excellent convective heat transfer effect.

Further, for a more comprehensive comparison of the convective heat dissipation of water and Ga-based liquid metal, researchers compared and calculated the convective heat transfer coefficients of Ga-based liquid metal and water under different operating conditions (different Reynolds numbers). This ratio of heat transfer coefficient between Ga-based liquid metal and water can well reflect the ability of different convective heat transfer media, which can be obtained based on the Table 2. For fully developed heat transfer in a circular pipe under constant heat flux condition, the ratio of heat transfer coefficient of water cooling and liquid metal cooling is listed as follows:

$$
\begin{gathered}
\frac{h_{l m}}{h_{\text {water }}}=\frac{h_{l m}}{h_{\text {water }}} \text { laminar flow } \\
\frac{h_{l m}}{h_{\text {water }}}=\frac{h_{l m}}{h_{\text {water }}} \frac{4.82+0.0185(\operatorname{RePr})^{0.827}}{0.023 \operatorname{Re}^{0.8} \operatorname{Pr}^{0.4}} \text { turbulent flow }
\end{gathered}
$$

Similarly, the ratio for constant temperature condition can also be obtained. As a result, the variation of the ratio $h_{l n} / h_{\text {water }}$ under different $R e$ numbers is illustrated in Fig. 5. It can be clearly seen that under laminar flow condition the value of $h_{l m} / h_{\text {water }}$ exceeds 60 , which indicates that the cooling capability of liquid metal is much superior than that of water. However, under turbulent flow condition, this advantage will largely decrease due to the fact that the heat transfer of water is significantly enhanced due to turbulence.

As a conclusion, the advantages of Ga-based liquid metal as convective media can be summarized as follows:

i. Non-toxic, Non-flammable, environmentally friendly;

ii. High thermal conductivity, which is dozens of times larger than that of water; iii. Excellent liquidity, the viscosity is the same magnitude as the viscosity of water;

iv. Adjustable physical properties, including doping, endocytosis, oxidation, etc.;

v. Excellent electrical conductivity and large surface tension lead to more driving methods;

vi. Low melting point and high boiling point ensure a wide liquid temperature zone;

vii. Electromagnetic pump drive, quiet, no moving parts.

\section{Applications on convective heat transfer}

As stated above, Ga-based liquid metal has been regarded as a kind of excellent heat transfer medium due to its superb physical and chemical properties. For example, the thermal conductivity of Ga-based liquid metals is generally $10 \sim 40 \mathrm{~W} /(\mathrm{m} \cdot \mathrm{K})$, two orders of magnitude higher than traditional cooling fluid (e.g. water). Such physical characteristic endows Ga-based liquid metal excellent convective heat transfer capacity. The replacement of traditional water-representative cooling fluids by Ga-based liquid metal is expected to break the limits of traditional cooling technology and provides new ideas for heat capture and transmission in other energy fields.

An increasingly accepted view is that liquid metal cooling technology has opened up a new era of next-generation highperformance cooling technology. Here, in order to demonstrate the academic progress and practical applications of Ga-based liquid metal in the field of convection heat dissipation, we show the heat dissipation performance of Ga-based liquid metal in three cases, including microchannel heat dissipation, two-phase flow heat dissipation and new cooling methods based on physical effects of liquid metal.

\subsection{Microchannel heat dissipation}

The appearance of the channel heat exchanger makes the dispersion device more compact, which reduces the amount of heat exchange medium and increases the heat transfer coefficient. In recent years, the microchannel heat transfer technology that has been widely concerned has made a series of progress. To enhance convective heat transfer efficiency in microchannel cooling for dissipating a large amount of

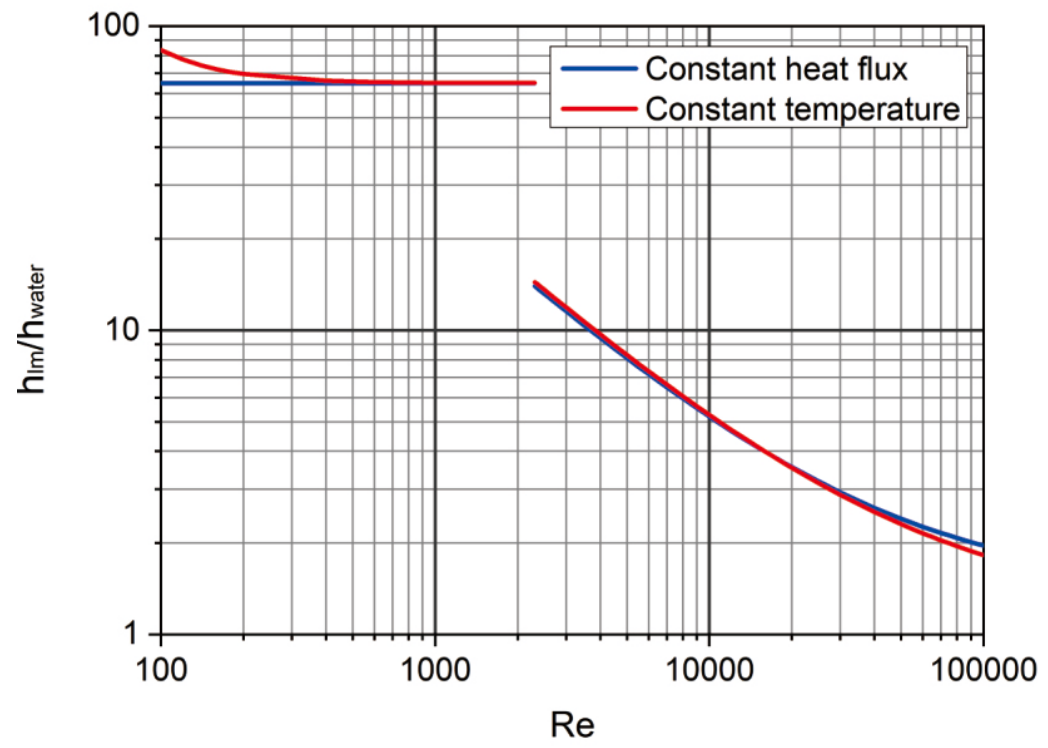

Fig. 5 Ratio of heat transfer coefficient of water and liquid metal $\left(\mathrm{Ga}_{68} \mathrm{In}_{20} \mathrm{Sn}_{12}\right)$ versus Re number." Here, the liquid metal mentioned refers to $\mathrm{Ga}_{68} \mathrm{In}_{20} \mathrm{Sn}_{12}$. Reproduced from Ref. 11, with permission from Elsevier. 
heat load as quickly as possible, researchers have proposed a series of methods, which can be summarized as structural optimization and surface modification. To be honest, the strategy of optimizing the structure and modifying the surface of microchannel is effective in many cases. Even so, it is vital to find a convective heat transfer medium with a better convective heat transfer coefficient. As stated before, Ga-based liquid metal is such a fluid that can efficiently exchange heat in the microchannel.
For the convective heat transfer, driving method is an important factor affecting the whole cooling system. Ga-based liquid metal is an excellent conductor of electricity and can be driven by electromagnetics, which is quite different from traditional working fluids driven by mechanical pumps. The principle of electromagnetic pump is shown in Fig. 6a. The $\mathrm{Z}$ reverse current is applied to the liquid metal, and the electromagnetic pump from the lower side is set in the $\mathrm{Y}$ direction. Then, the driving force is generated perpendicular to the current and the a

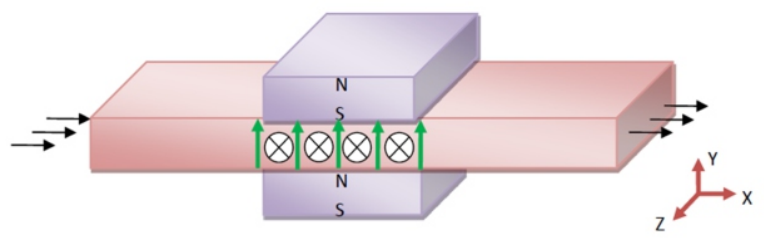

b

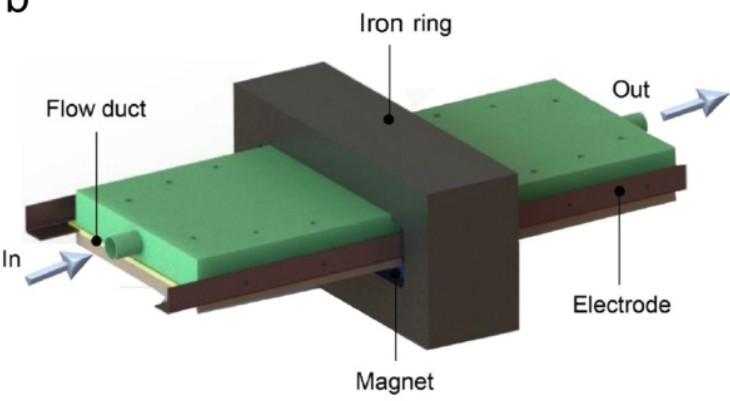

Fig. 6 Electromagnetic pump for driving liquid metal. a) Schematic diagram of electromagnetic pump. b) The specific structure of the electromagnetic pump. Reproduced from Ref. 79, with permission from Elsevier.
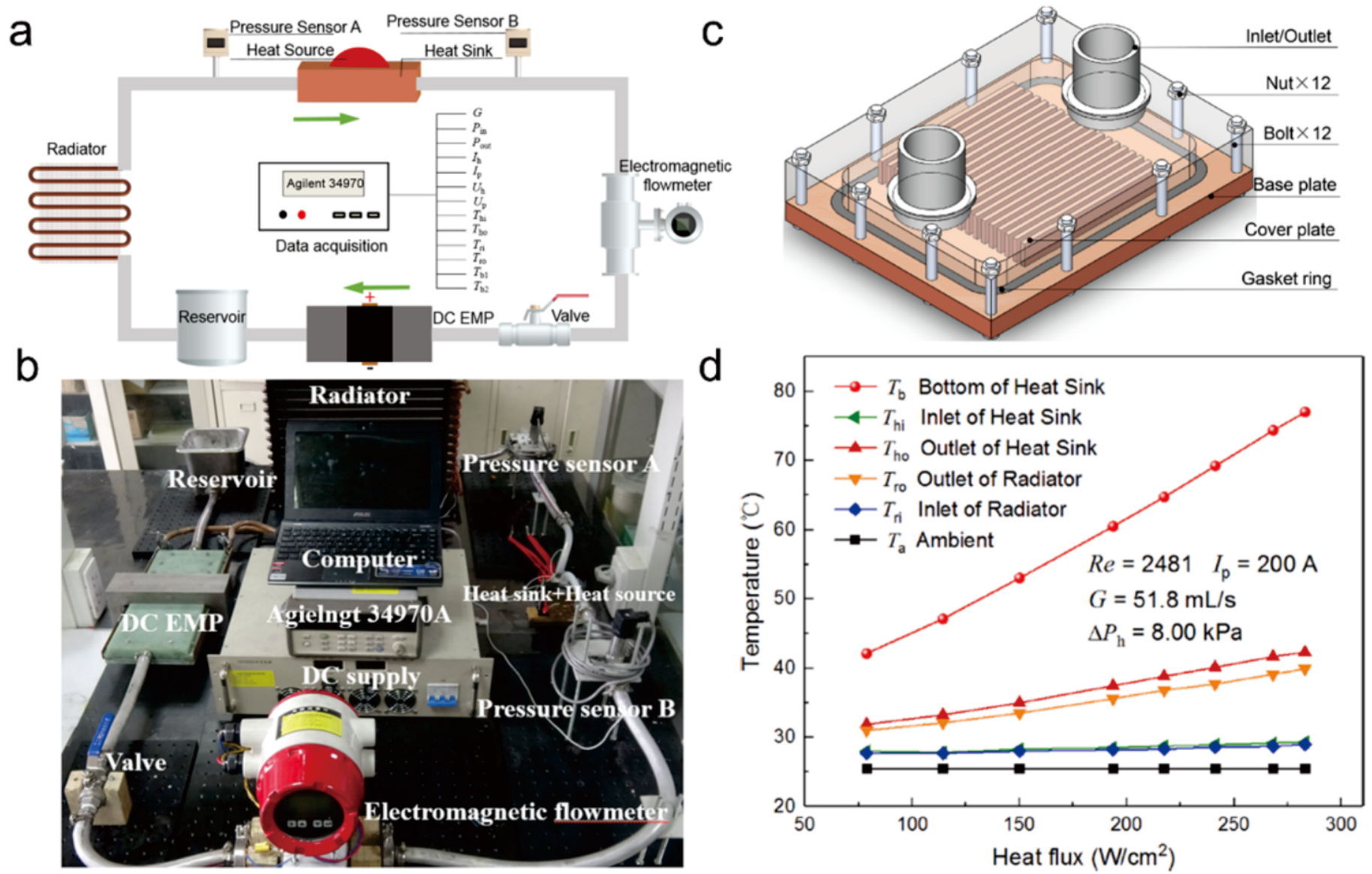

Fig. 7 a) The schematic diagram of the test loop. b) The experimental loop. c) The structure of mini-channel heat sink. d) The temperature of Ga-based liquid metal $\left(\mathrm{Ga}_{68} \mathrm{In}_{20} \mathrm{Sn}_{12}\right)$ based mini-channel cooling system under different heat fluxes with pumping currents 200 A. Here, $G$ is the volume flow rate, $P_{\mathrm{hi}}$ is the inlet pressure of heat sink, $P_{\mathrm{ho}}$ stands for the outlet pressure of heat sink, $I_{\mathrm{h}}$ is the heating current of heat source and $I_{\mathrm{p}}$ represents the pumping current of electromagnetic pump. ${ }^{79}$ Reproduced from Ref. 79, with permission from Elsevier. 
magnetic field, which is used to drive liquid metal movement. Next, the liquid metal flows in the $\mathrm{X}$ direction. In addition, because liquid metal has a high electrical conductivity, a large current can be generated by applying a small voltage, which is conducive to practical applications. The specific structure of the electromagnetic pump is illustrated in Fig. 6b. Since the electromagnetic pump has no moving parts, it owns the advantages of being relatively reliable and having no noise. Therefore, the use of electromagnetic pumps to drive liquid metal for convective cooling has a unique advantage.

Further, researchers experimentally investigated the Ga-based liquid metal mini-channel cooling for extremely high heat flux. ${ }^{79}$ Here, the electromagnetic pump for effectively driving Ga-based liquid metal was proposed, which can provide high pressure head up to $100 \mathrm{kPa}$. Experimental results indicated that the cooling system driven by electromagnetic pump can operate silently, steadily and smoothly because of the fact that there are no active parts in the system. The schematic diagram of the experimental loop is shown in Fig. 7a. Then, as illustrated in Fig. $7 b$, the researchers built the actual thermal test device. The structure of designed and manufactured mini-channel heat sink with width of channel $1 \mathrm{~mm}$, height of channel $5 \mathrm{~mm}$ was displayed in Fig. 7c. The experiment results show that Ga-based liquid metal mini-channel cooling system can dissipate heat with heat flux 300 $\mathrm{W} / \mathrm{cm}^{2}$ (Figure 7d). Compared to water mini-channel cooling, Ga-based liquid metal mini-channel cooling can obtain a rather larger heat transfer enhancement, which indicates its excellent convection cooling effect. As a summary, the liquid metal mini-channel cooling can be regarded to be an effective and practical way for the thermal management of high heat flux, especially for those situations where extreme heat flux exists.

\subsection{Two-phase flow cooling}

As the most classical coolant in a convective heat transfer process, water has been widely adopted which however inherits with limited thermal conductivity and relies heavily on mechanical pump. As an alternative, the Ga-based liquid metal is increasingly emerging as an important coolant to realize much stronger enhanced heat transfer. However, its thermal capacity is somewhat lower than that of water, which may restrict the overall cooling performance. In addition, the high cost by taking too much amount of Ga-based liquid metal into the device also turns out to be a big cost concern for practical purpose. Therefore, in order to solve this problem, researchers proposed a new conceptual liquid metal cooling strategy, that is, two-phase flow cooling by combining the individual merits from both Ga-based liquid metal with high thermal conductivity and water with large specific heat capacity.

As discussed above, electric field enables the Ga-based liquid metal to be quickly accelerated to a large speed under only a low electric voltage, which can be used to drive a Ga-based liquid metal cooling medium for heat dissipation. The electrically induced actuation effect of Ga-based liquid metal will be introduced as the flow driving strategy, which significantly simplified the whole system design. With this unique property, researchers can avoid the use of electromagnetic pumps, but adopt the power supply directly to drive liquid metal for heat dissipation. Tan et al. ${ }^{62}$ constructed an electrically driven chip cooling device using hybrid coolants of Ga-based liquid metal and water-based

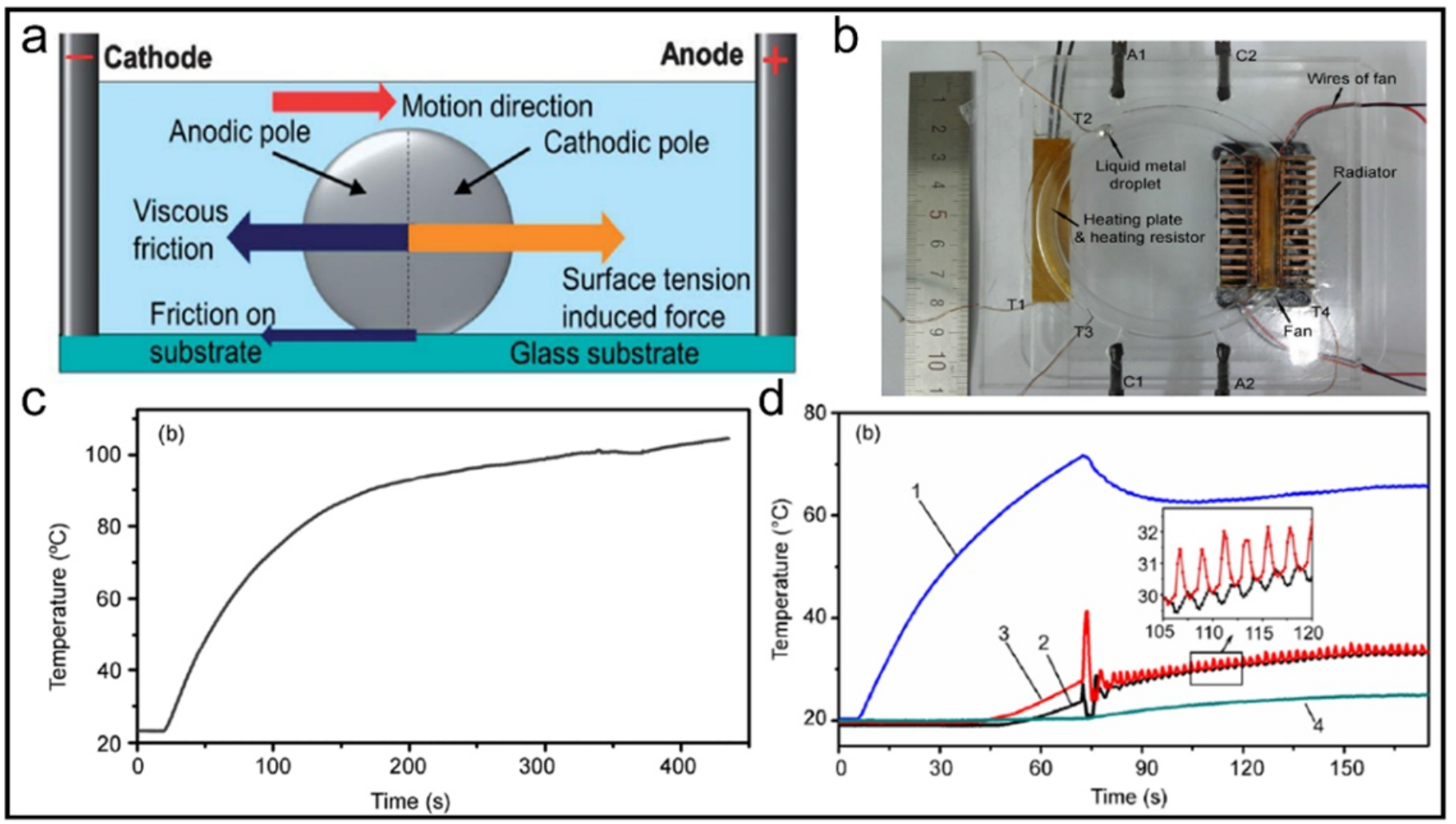

Fig. 8 Electrically driven chip cooling device using hybrid coolants of liquid metal and aqueous solution. a) Schematic diagram of forces influencing the motion of liquid metal droplets placed in the electric flied. ${ }^{58}$ Reproduced from Ref. 58, with permission from Royal Society of Chemistry. b) Working prototype of the hybrid coolant cooling device. Here, T1-T4 represent thermocouples. A1/C1, A2/C2 are two pairs of electrodes. c) Temperature transients of the heating plate under different $8 \mathrm{~W}$ power without metal droplet cooling. d) Temperature transients of the device under $8 \mathrm{~W}$ heating powers. Here, 1 stands for heating plate; 2 stands for inlet of heating plate; 3 stands for outlet of heating plate; 4 , radiator fins. ${ }^{62}$ Reproduced from Ref. 62 , with permission from springer. 
aqueous solution. As illustrated in Fig. 8a, Ga-based liquid metal immersed in electrolyte solution is subjected to multiple forces and moves toward the positive electrode by applying an electric field. Research disclosed that the linear speed of Ga-based liquid metal can be easily accelerated to a high value $(8 \mathrm{~cm} / \mathrm{s})$ under a small voltage.

To test the feasibility of the system, working prototype of the hybrid coolant cooling device was constructed (Fig. 8b). The heat transfer performance of the device is systematically explored under several different heat fluxes. The results of the test show that the temperature of the hot plate will rise continuously without the cooling of the liquid metal droplets (Fig. 8c). Conversely, the temperature of the hot plate can be effectively controlled by utilizing liquid metal droplets (Fig. 8d). Compared with other traditional convective heat transfer system, the cooling system takes a highly compact structure, which can be flexibly applied in a wide variety of application scenario. More importantly, such new conceptual cooling method based on electric field driven Ga-based liquid metal can avoid consuming more liquid metal and is beneficial to the economics of the overall cooling system.

In addition to the two-phase flow of water and Ga-based liquid metal, the two-phase flow between the low-boiling working medium (isopentane) and the Ga-based liquid metal for heat transfer is also studied. A close-cycle self-driving thermal energy harvester using Gabased liquid metal as energy carrier fluid has been proposed. ${ }^{80}$ The driving force that pushes the Ga-based liquid metal against flow resistance and gravity is provided by a resistively heated low-bowing fluid based on thermo-pneumatic principle. The cyclical operating process of the harvester prototype is shown in Fig. 9a. Here, the gravitational force drove the Ga-based liquid metal and the condensed isopentane back to the heat source chamber, thus leading to the result that the system will return to its initial state (Fig. 2b and Fig. 2c). Further research reveals a stable liquid metal recirculation performance with a time average volume flow of $14 \mathrm{ml} / \mathrm{min}$ and a heating load of 12.25 watts can be achieved during the 5 -hour operation. The prototype also managed to adjust itself to variable working conditions, which demonstrates the reliability of this approach. Advantages of the method include simple structural design, no rigid motion operation and low temperature actuation, which makes it particularly suitable for solar energy and low-level heat collection, high heat flux electronic cooling, etc.

\subsection{New cooling method based on physical effects of liquid metal} Liquid metal, as an untraditional fluid, owns the largest surface tension among all the room temperature fluid and displays rather rich hydrodynamic phenomena. By taking advantage of these hydrodynamic phenomena, researchers have investigated a group of novel methods to drive the liquid metal for heat dissipation. Here, the thermal induced

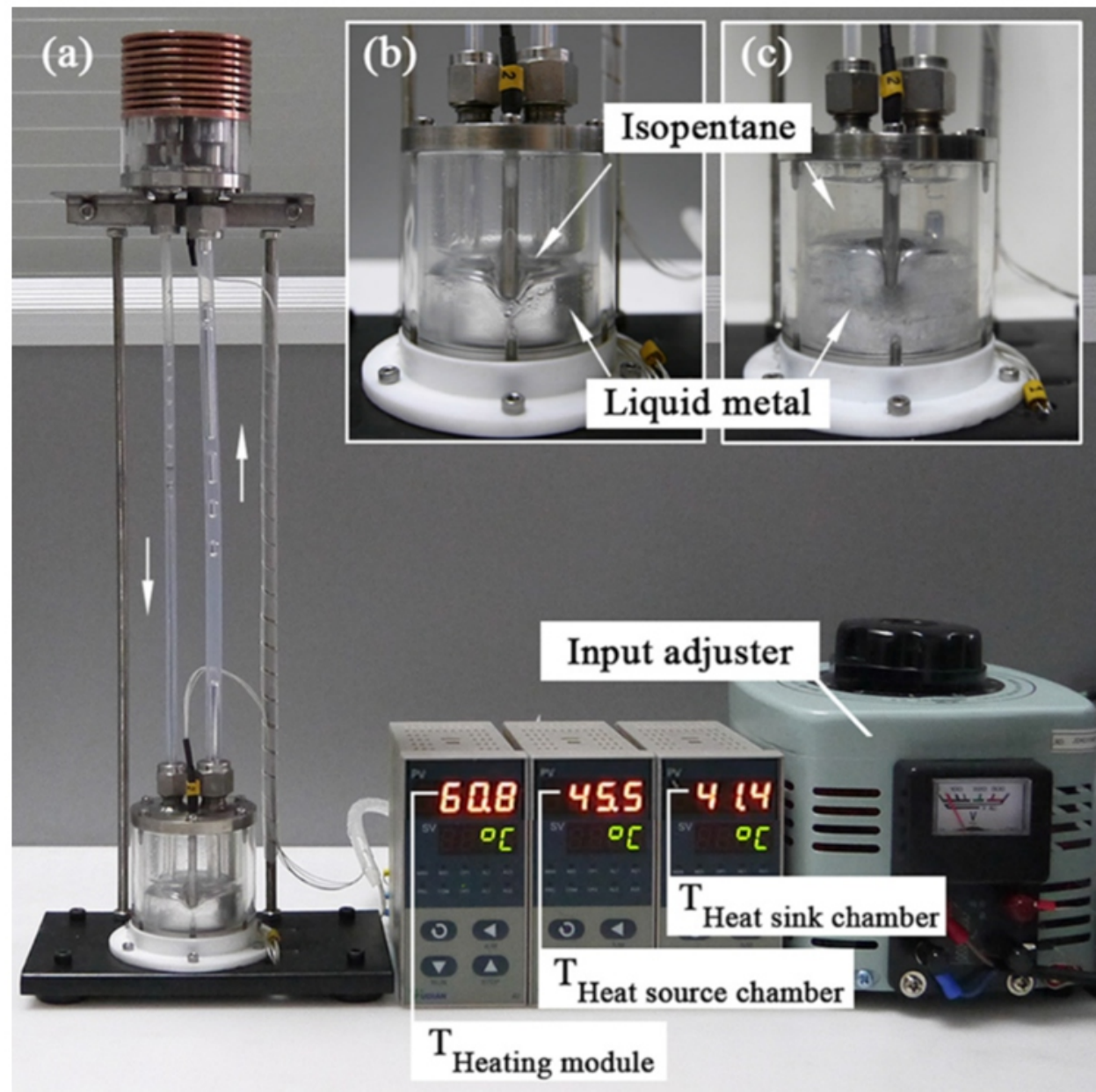

Fig. 9 a) Experimental set-ups of the tested harvester prototype showing its working process with $6.25 \mathrm{~W}$ heating power. b) Liquid metal and isopentane distribution in the heat source chamber during operation. c) Liquid metal and isopentane layered in the heat source chamber $1 \mathrm{~h}$ after heat charge is terminated. ${ }^{80}$ Reproduced from Ref. 80, with permission from American Institute of Physics. 
thermosiphon effect and the liquid metal spin motion effect excited by electric field will be introduced separately.

Li et al. study, driving Ga-based liquid metal under thermosiphon effect for electronic cooling was demonstrated. ${ }^{63}$ The working principle and prototype are illustrated in Fig. 10. In this system, the buoyancy force will be induced by the external heat, which in turn drives the Gabased liquid metal. As a result, flowing liquid metal can effectively remove heat from the heat source. Experimental results indicate that such thermal management strategy can stably keep the temperature of the heat source at $87.7^{\circ} \mathrm{C}$ without any extra fan working when the heat load is $42.1 \mathrm{~W}$. For various reasons, the results obtained in the current principle experiments are more to demonstrate the effectiveness of this strategy, which is far from optimal performance. The cooling capacity of this system is closely related to the heat source intensity and height difference, so that the greater temperature difference and the improved height difference can significantly improve the cooling capacity of this system. In addition, the cooling performance of the method can be further significantly improved by doping other particles to improve the properties of the Ga-based liquid metal. The thermosiphon effect driving liquid metal to realize the heat dissipation cooling strategy has the advantages of self-driving, simple structure, high efficiency cooling, energy saving, low noise, etc., showing excellent application prospects.

In addition, Ga-based liquid metal itself can be used as a pump to drive coolant medium by applying an electric field. The corresponding research was also carried out. ${ }^{61}$ Researchers found that the Ga-based liquid meal droplet fixed in the copper tale will rotate continuously by applying square wave DC signal. Marangoni flow is generated due to the continuous electrowetting effect at the surface of the liquid metal droplet with the application of electric signal, ${ }^{81}$ which can be used to drive the coolant medium through the designed channel for heat dissipation (Fig. 11a). Besides, as illustrated in Fig. 11a (ii), Ga-based liquid metal droplet also serves as an effective heat sink because of its high thermal conductivity, conducting heat into the coolant medium.
Further, as shown in Fig. 11b, researchers applied infrared cameras to demonstrate the results of the heat dissipation, indicating that effective convection cooling has been achieved. Quantitatively, the temperature at the hot plate was also collected, confirming that the temperature has indeed been reasonably controlled (Fig. 11c). To enhance the convective cooling coefficient, fluids with better thermal conductivity can also be driven by utilizing the Ga-based liquid metal pump. More importantly, the flow rate of the cooling medium can be readily tuned by varying the voltage and frequency of the applied DC signal. Also, eliminating external connections and moving parts reduces the response time of the cooling system based on liquid metal pump, which enables fast cooling of the electronic chip. The simple and customizable experimental setup also facilitates the thermal management of electronic chips with high power density and limited space.

Up to now, one can draw a conclusion that the recently applied liquid metal cooling strategy is expected to pave the way for high flux heat dissipation, which is difficult to tackle otherwise by existing conventional cooling methods. However, the application of thermal management technology is ever-changing, and the working environment and requirements are complicated. In order to fundamentally promote the establishment of more advanced cooling technologies, researchers systematically proposed the concept of "composite heat transfer based on liquid metal". ${ }^{81}$ The basic idea of "composite heat transfer based on liquid metal" is to modularize the heat dissipation system, and each module has its own basic heat transfer mode and characteristics. In general, a conventional cooling system can be abstractly divided into five parts: heat source, heat extraction, heat transfer, heat release, and the surrounding environment. Depending on the application, different modules can be organically combined to form a specific cooling system to meet specific requirements with optimum performance. Advanced liquid metal cooling technologies, including high-performance thermal interface materials, thermal spreaders, convection cooling, two-fluid cooling, phase change thermal control and energy storage, can play
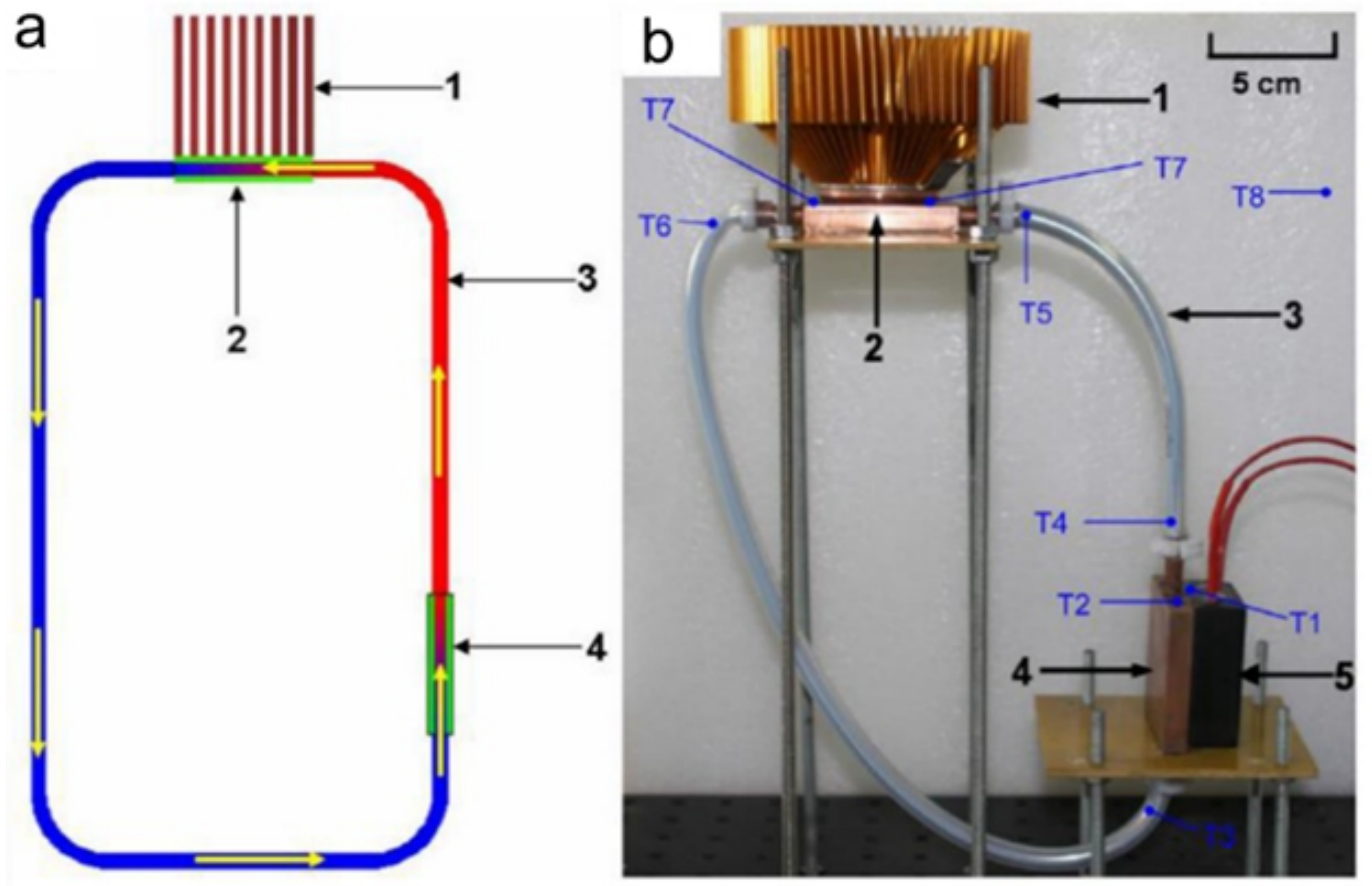

Fig. 10 Thermosyphon effect of liquid metal enabled passive cooling system. a) Working principle; b) prototype..$^{63}$ Reproduced from Ref. 63 , with permission from the American Society of Mechanical Engineers. 
different roles in different modules. By combining liquid metal cooling technology with traditional cooling technology, applying it to the most needed heat transfer modules, solving the heat transfer bottleneck of key links, it is possible to construct an optimal cooling strategy and achieve better cooling capacity and value for money.

\section{Conclusions and outlooks}

In conclusion, we have summarized the properties of Ga-based liquid metal as a fluid and its applications in convective heat transfer. Besides, strategies to improve the properties of Ga-based liquid metal are also presented and the corresponding basic theory of liquid metal heat transfer is discussed. The recently emerging efforts of enabling Gabased liquid metals as unconventional convective heat transfer medium have been systematically reviewed. So far, the commonly tried Gabased liquid metals are gallium indium eutectic alloy $\left(\mathrm{GaIn}_{24.5}\right)$ and gallium indium tin eutectic alloy $\left(\mathrm{Ga}_{67} \mathrm{In}_{21} \mathrm{Sn}_{12}\right)$. It is now clear that these metals would provide valuable insights associated with heat transfer which are essential for future industrial research and applications. Apart from the low melting point, Ga-based liquid metal exhibits superior and fundamental advantages in flow features, thermal and electrical conductivity, plasticity, non-flammability, and biocompatibility, compared with mercury and traditional solid metals. Such unique characteristics enable Ga-based liquid metal to be potentially developed as future extraordinary heat transfer medium.

The relevant studies are advancing so rapidly that they have been gradually embedded in a wide variety of emerging applications. It is believed that liquid metal as heat transfer medium will be sure to contribute for heat transfer. Meanwhile, such unconventional liquids also raised interesting and important issues waiting for clarification and improvement in the near future. In order to significantly extend future industrial practices of these materials, tremendous efforts are required to better understand their instinct properties and long-term performances, aiming to guarantee a systematic assessment of the new application. Though Ga-based liquid metal with a high thermal conductivity has been studied a lot, some challenges still exist. Herein, we list some of them to guide future practices in the field.

I. In many cases, melting points of liquid metal restrict its application. Therefore, new types of Ga-based liquid metal are urgently needed, especially those whose melting points are below $0{ }^{\circ} \mathrm{C}$ or even lower. Alloying is still a direction worth exploring.

II. Enhance the physical properties of Ga-based liquid metal will be necessary. Here, physical properties include but are not limited to thermal conductivity, specific thermal capacity, etc. Doping by other metal particles or non-metal particles reserves to be a very important method and more effective doping strategies need to be discovered.

III. Mature driving mode of liquid metal in micro flow channels, corrosion problems for the long-time application and adhesion problems due to oxidation are all needed to be solved for the practical use of liquid metal convection cooling technology.

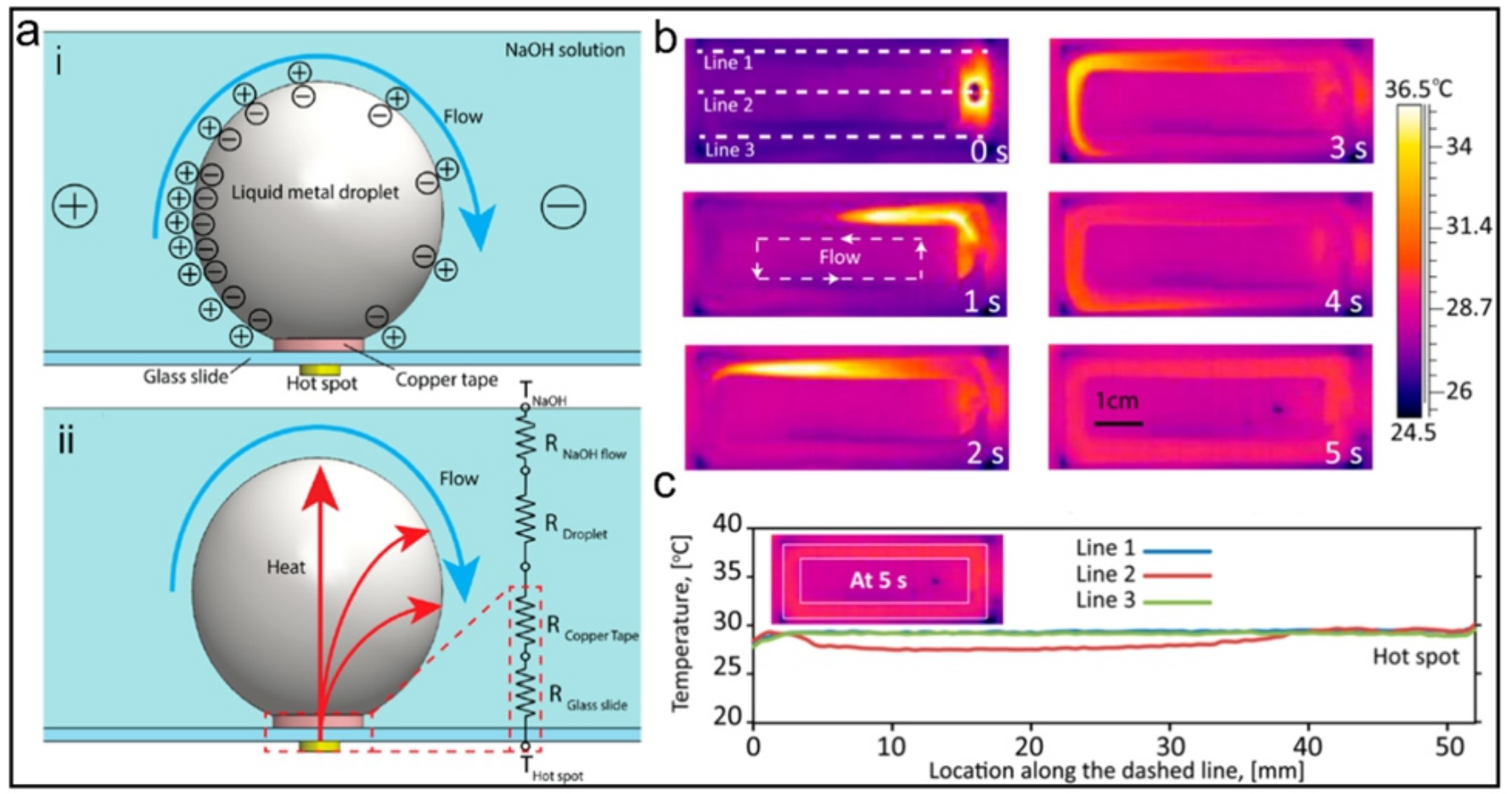

Fig. 11 An integrated liquid cooling system based on liquid metal droplets. a) Operation mechanism of actuated liquid metal over the hot spot. i Schematic diagram of liquid metal droplet surface charge distribution when an electric potential is applied. ii Schematic and equivalent thermal circuit of heat dissipation through liquid metal droplet, where $\mathrm{T}$ is temperature and $\mathrm{R}$ is thermal resistance. b) Sequential snapshots obtained by infrared camera showing the variations of temperature in space with respect to time. c) Temperature distribution after actuating the liquid metal. Results are obtained by using a $3 \mathrm{~mm}$ liquid metal droplet $\left(\mathrm{Ga}_{67} \mathrm{In}_{21} \mathrm{Sn}_{12}\right)$ immersed in $0.3 \mathrm{M} \mathrm{NaOH}$ solution and actuated with a square wave signal $(200 \mathrm{~Hz}, 5 \mathrm{Vp}-\mathrm{p}, 2.5 \mathrm{~V}$ dc offset and 50\% duty cycle). The microheater is energized with a $3.5 \mathrm{~V}, 0.04 \mathrm{~A}$ dc signal. ${ }^{61}$ Reproduced from Ref. 61, with permission from American Chemical Society. 
IV. Reducing the economic cost of a heat transfer system is an eternal theme. Combining liquid metal with other heat transfer media (such as water) is expected to reduce costs and more research work needs to be carried out along this way.

\section{Conflict of interest}

There are no conflicts to declare.

\section{Acknowledgements}

This work is partially supported by NSFC Key Project under Grant No. 91748206, Dean's Research Funding and the Frontier Project of the Chinese Academy of Sciences.

\section{References}

1. S. M. S. Murshed and C. A. N. D. Castro, Renew. Sust. Energ. Rev., 2017, 78, 821-833.

2. Y. W. Chang, C. C. Chang, M. T. Ke and S. L. Chen, Appl. Therm. Eng., 2009, 29, 2731-2737.

3. C. J. Zhao, J. W. Han, X. T. Yang, J. P. Qian and B. L. Fan, Appl. Energ., 2016, 168, 314-331.

4. K. S. Kim, M. H. Won, J. W. Kim and B. J. Back, Appl. Therm. Eng., 2003, 23, 1137-1144.

5. D. Khrustalev and A. Faghri, J. Heat Transfer, 1994, 116, 189-198.

6. A. H. Khalaj and S. K. Halgamuge, Appl. Energ., 2017, 205, 1165-1188.

7. H. Wang, Z. Chen and J. Gao, Appl. Therm. Eng., 2016, 107, 870-879.

8. F. Yang, X. Dai, Y. Peles, P. Cheng, J. Khan and C. Li, Int. J. Heat Mass Transfer, 2014, 68, 703-715.

9. H. M. S. Bahaidarah, Sol. Energy, 2016, 135, 605-617.

10. T. Li, Y. G. Lv, J. Liu and Y. X. Zhou, Forsch Ingenieurwes, 2006, 70, 243-251.

11. X. H. Yang and J. Liu, Adv. Heat Transfer, 2018, 50, 187-300.

12. A. Miner and U. Ghoshal, Appl. Phys. Lett., 2004, 85, 506-508.

13. Y. Deng and J. Liu, Int. Commun. Heat Mass, 2010, 37, 788-791.

14. K. Ma and J. Liu, Front. Energy, 2007, 1, 384-402.

15. A. Zrodnikov, A. Efanov, Y. I. Orlov, P. Martynov, V. Troyanov and A. Rusanov, At. Energ., 2004, 97, 534-537.

16. K. Mikityuk, Nucl. Eng. Des., 2009, 239,680-687.

17. R. P. Stein, Adv. Heat Transfer, 1966, 3, 101-174.

18. X. H. Yang, S. C. Tan and J. Liu, Int. J. Heat Mass Transfer, 2016, 100, 899-907.

19. I. A. de Castro, A. F. Chrirnes, A. Zavabeti, K. J. Berean, B. J. Carey, J. Zhuang, Y. Du, S. X. Dou, K. Suzuki, R. A. Shanks, R. Nixon-Luke, G. Bryant, K. Khoshmanesh, K. Kalantar-zadeh and T. Daeneke, Nano Lett., 2017, 17, 7831-7838.

20. X. H. Yang, S. C. Tan, Z. Z. He and J. Liu, Energy Convers. Manage., 2018, 160, $467-476$

21. X. L. Wang and J. Liu, Micromachines., 2016, 7, 206,

22. R. Guo, H. Z. Wang, M. H. Duan, W. Z. Yu, X. J. Wang and J. Liu, Smart Mater. Struct. 2018, 27, 085022.

23. N. Ilyas, A. Cook and C. E. Tabor, Adv. Mater. Interfaces, 2017, 4, 1700141.

24. Q. Wang, Y. Yu, J. Yang and J. Liu, Adv. Mater., 2015, 27,7109.

25. N. Ilyas, A. Cook and C. E. Tabor, Adv. Mater. Interfaces, 2017, 4, 1700141.

26. X. Wang, W. Yao, R. Guo, X. Yang, J. Tang, J. Zhang, W. Gao, V. Timchenko and J. Liu, Adv Healthc Mater, 2018, 7, 085022.

27. S. A. Chechetka, Y. Yu, X. Zhen, M. Pramanik, K. Y. Pu and E. Miyako, Nat. Commun., 2017, 8, 19.

28. C. Jin, J. Zhang, X. Li, X. Yang, J. Li and J. Liu, Sci. Rep., 2013, 3, 3442.

29. J. Yan, Y. Lu, G. Chen, M. Yang and Z. Gu, Chem. Soc. Rev., 2018, 47, 2518-2533.

30. L. T. Yi and J. Liu, Int. Mater. Rev., 2017, 62, 415-440.
31. T. Daeneke, K. Khoshmanesh, N. Mahmood, I. A. de Castro, D. Esrafilzadeh, S. J. Barrow, M. D. Dickey and K. Kalantar-zadeh, Chem. Soc. Rev., 2018, 47, 4073-4111.

32. B. Lertanantawong, J. D. Riches and A. P. O' Mullane, Langmuir, 2018, 34,7604-7611.

33. A. Zavabeti, J. Z. Ou, B. J. Carey, N. Syed, R. Orrelltrigg, E. Mayes, C. Xu, O. Kavehei, A. P. O'Mullane and R. B. Kaner, Science, 2017, 358, 332-335.

34. D. Esrafilzadeh, A. Zavabeti, R. Jalili, P. Atkin, J. Choi, B. J. Carey, R Brkljaca, A. P. O'Mullane, M. D. Dickey, D. L. Officer, D. R. MacFarlane, T. Daeneke and K. Kalantar-Zadeh, Nat. Commun., 2019, 10, 865.

35. S. Chen, X. Yang, Y. Cui and J. Liu, ACS Appl. Mater. Inter., 2018, 10 22889-22895

36. J. Zhang, Y. Y. Yao, L. Sheng and J. Liu, Adv. Mater, 2015, 27, 2648-2655.

37. B. Yuan, S. Tan, Y. Zhou and J. Liu, Sci. Bull., 2015, 60, 1203-1210.

38. J. Liu, L. Sheng and Z. Z. He, Liquid Metal Soft Machines, 2018, Springer.

39. T. Y. Liu, P. Sen and C. Kim, J. Microelectromech. S., 2012, 21, 443-450.

40. X. D. Zhang, Y. Sun, S. Chen and J. Liu, Front. Energy,_2018, 12, 276-296.

41. J. Liu, L. Yi, Liquid Metal Biomaterials, 2018, Springer.

42. Y. Z. Yu, J. R. Lu and J. Liu, Mater. Design, 2017, 122, 80-89.

43. G. Bo, L. Ren, X. Xu, Y. Du and S. Dou, Adv. Physics-X, 2018, 3, 1446359.

44. F. J. Shaini, R. M. Shelton, P. M. Marquis and A. C. Shortall, Biomaterials, 2000, 21,113-119.

45. V. Psarras, A. Wennberg and T. Dérand, Acta Odontol. Scand., 1992, 50, 31-36.

46. A. Milheiro, K. Nozaki, C. J. Kleverlaan, J. Muris, H. Miura and A. J. Feilzer, Odontology, 2016, 104, 136-142.

47. J. H. Kim, S. Kim, J. H. So, K. Kim and H. J. Koo, ACS Appl. Mater. Interfaces, 2018, 10, 17448-17454.

48. H. W. Chen, B. Environ. Contam. Tox., 2007, 78, 113-117.

49. K. L. Chang, W. T. Liao, C. L. Yu, C. C. E. Lan, L. W. Chang and H. S. Yu, Toxicol. Appl. Pharm., 2003, 193, 209-217.

50. J. E. Chandler, H. H. Messer and G. Ellender, J. Dent. Res., 1994, 73, 1554.

51. D. I. U. M€uller, D. I. L. B€uhler, Springer, Berlin, Heidelberg, 2001.

52. Y. Deng and J. Liu, Heat Mass Transfer, 2010, 46,1327-1334.

53. B. Yuan, L. Wang, X. H. Yang, Y. J. Ding, S. C. Tan, L. T. Yi, Z. Z. He and J. Liu, Adv. Sci., 2016, 3, 4.

54. S. Y. Tang, K. Khoshmanesh, V. Sivan, P. Petersen, A. P. O'Mullane, D Abbott, A. Mitchell and K. Kalantar-Zadeh, Proc. Natl. Acad. Sci. U.S.A. 2014, 111, 3304.

55. S. C. Tan, X. H. Yang, H. Gui, Y. J. Ding, L. Wang, B. Yuan and J. Liu, Soft Matter, 2017, 13, 2309-2314.

56. Z. Ali, D. Torben, A. F. Chrimes, A. P. O'Mullane, Z. O. Jian, M. Arnan, K. Khashayar and K. Z. Kourosh, Nat. Commun., 2016, 7,12402.

57. L. Sheng, J. Zhang and J. Liu, Adv. Mater, 2014, 26, 6036-6042.

58. S. Y. Tang, V. Sivan, K. Khoshmanesh, A. P. O'Mullane, X. Tang, B. Gol, N. Eshtiaghi, F. Lieder, P. Petersen and A. Mitchell, Nanoscale, 2013, 5, 59495957.

59. L. Sheng, J. Zhang and J. Liu, Adv. Mater., 2014, 26, 6036-6042.

60. A. Zavabeti, T. Daeneke, A. F. Chrimes, A. P. O'Mullane, J. Z. Ou, A. Mitchell, K. Khoshmanesh and K. Kalantar-zadeh, Nat. Commun., 2016, 7, 12402 .

61. J. Y. Zhu, S. Y. Tang, K. Khoshmanesh and K. Ghorbani, ACS Appl. Mater. Inter, 2016, 8, 2173-2180.

62. S. Tan, Y. Zhou, L. Wang and J. Liu, Sci. China Technol. Sci., 2016, 59, 301-308.

63. P. Li and J. Liu, J. Electron. Packaging, 2011, 133, 041009.

64. X. Zhao, J. Tang and J. Liu, Phys. Rev. Fluids, 2018, 3, 124804.

65. F. Carle, K. Bai, J. Casara, K. Vanderlick and E. Brown, Phys. Rev. Fluids, 2017, 2, 013301

66. M. J. Assael, K. Kakosimos, R. M. Banish, J. Brillo, I. Egry, R. Brooks, P. N. Quested, K. C. Mills, A. Nagashima and Y. Sato, J. Phys. Chem. Ref. Data, 2006, 35, 285-300

67. M. Jeyakumar, M. Hamed and S. Shankar, J. Non-Newton. Fluid., 2011, 166, 831-838

68. E. Ricci, E. Arato, A. Passerone and P. Costa, Adv. Colloid Interface Sci. 
2005, 117, 15-32.

69. H. Y. Li, S. F. Mei, L. Wang, Y. X. Gao, and J. Liu, Int. J. Heat and Fluid Flow, 2014, 47, 1-8.

70. Q. Xu, N. Oudalov, Q. T. Guo, H. M. Jaeger and E. Brown, Phys. Fluids, 2012, 24, 063101.

71. J. Tang, X. Zhao, J. Li, R. Guo, Y. Zhou and J. Liu, ACS Appl. Mater. Inter, 2017, 9, 35977-35987.

72. X. L. Wang, W. H. Yao, R. Guo, X. H. Yang, J. B. Tang, J. Zhang, W. P. Gao, V. Timchenko and J. Liu, Adv. Health. Mater., 2018, 7, 1800318.

73. H. Wang, B. Yuan, S. Liang, R. Guo, W. Rao, X. Wang, H. Chang, Y. Ding, J. Liu and L. Wang, Mater. Horiz., 2018, 5, 222-229.

74. L. Ren, S. S. Sun, G. Casillas-Garcia, M. Nancarrow, G. Peleckis, M. Turdy, K. R. Du, X. Xu, W. H. Li, L. Jiang, S. X. Dou and Y. Du, Adv. Mater, 2018, 30, 9.

75. H. Chang, R. Guo, Z. Sun, H. Wang, Y. Hou, Q. Wang, W. Rao and J. Liu,
Adv. Mater. Interfaces, 2018, 5, 1800571.

76. X. D. Zhang, Y. Sun, S. Chen and J. Liu, Front. Energy, 2018, 12, 276-296.

77. J. M. Coulson, J. F. Richardson, J. R. Backhurst and J. H. Harker, Filtr. Separat., 1996, 33, 102.

78. T. L. Bergman, F. P. Incropera, A. S. Lavine, Fundamentals of Heat and Mass Transfer, John Wiley \& Sons, 4th edn., 1966.

79. X. D. Zhang, X. H. Yang, Y. X. Zhou, W. Rao, J. Y. Gao, Y. J. Ding, Q. Q. Shu and J. Liu, Energy Convers. Manage., 2019, 185, 248-258.

80. J. Tang, J. Wang, J. Liu and Y. Zhou, Appl. Phys. Lett., 2016, 108, 023903.

81. S. Y. Tang, K. Khoshmanesh, V. Sivan, P. Petersen, A. P. O'Mullane, D. Abbott, A. Mitchell and K. Kalantar-Zadeh, Proc. Natl. Acad. Sci. U.S.A. 2014, 111, 3304

82. X. H. Yang and J. Liu, Front. Energy, 2018, 12, 259-275.

Publisher's Note Engineered Science Publisher remains neutral with regard to jurisdictional claims in published maps and institutional affiliations. 